\title{
Water wettability of close-packed metal surfaces
}

\section{Citation}

Meng, Sheng, Efthimios Kaxiras, and Zhenyu Zhang. 2007. "Water Wettability of ClosePacked Metal Surfaces." The Journal of Chemical Physics 127 (24): 244710. https:// doi.org/10.1063/1.2804871.

\section{Permanent link}

http://nrs.harvard.edu/urn-3:HUL.InstRepos:41384022

\section{Terms of Use}

This article was downloaded from Harvard University's DASH repository, and is made available under the terms and conditions applicable to Other Posted Material, as set forth at http:// nrs.harvard.edu/urn-3:HUL.InstRepos:dash.current.terms-of-use\#LAA

\section{Share Your Story}

The Harvard community has made this article openly available.

Please share how this access benefits you. Submit a story.

\section{Accessibility}




\title{
Water wettability of close-packed metal surfaces
}

\author{
Sheng Meng ${ }^{a)}$ \\ Department of Physics and School of Engineering and Applied Sciences, Harvard University, \\ Cambridge, Massachusetts 02138, USA, Department of Physics, University of Texas, \\ Austin, Texas 78712, USA, and Condensed Matter Sciences Division, Oak Ridge National Laboratory, \\ Oak Ridge, Tennessee 37831, USA \\ Efthimios Kaxiras \\ Department of Physics and School of Engineering and Applied Sciences, Harvard University, \\ Cambridge, Massachusetts 02138, USA \\ Zhenyu Zhang \\ Condensed Matter Sciences Division, Oak Ridge National Laboratory, Oak Ridge, Tennessee 37831, USA \\ and Department of Physics and Astronomy, University of Tennessee, \\ Knoxville, Tennessee 37996, USA
}

(Received 2 July 2007; accepted 9 October 2007; published online 28 December 2007)

\begin{abstract}
We propose a new microscopic criterion to determine surface wetting: water wets the surface whenever its overlayer has a larger adsorption energy than three-dimensional clusters on the bare or water-covered substrate. This conceptually intuitive criterion is validated by detailed first-principles calculations of the energetics of layers and clusters of water on different metal surfaces. This criterion resolves naturally the current discrepancy between theory and experiment on the wetting behavior of undissociated water on $\mathrm{Ru}(0001)$, as well as the hydrophobic nature of the $\mathrm{Au}(111)$ surface. It also explains the Stranski-Krastanov ice growth on $\mathrm{Pt}(111)$ observed experimentally.

(C) 2007 American Institute of Physics. [DOI: 10.1063/1.2804871]
\end{abstract}

Water wetting and ice growth on a surface are among the most common observations in nature; these processes are of fundamental importance in technological applications such as corrosion, catalysis, electrochemistry, and solar and fuel cells and even in biomedical applications, as is evidenced by the existence of antifreezing proteins. The competition between wetting and ice growth is partly due to the fact that hydrogen bonds ( $\mathrm{H}$ bonds) among neighboring water molecules have similar strength to bonds between water molecules and surface atoms for many common surfaces. This has inspired extensive experimental and theoretical studies of water wetting in recent decades, ${ }^{1,2}$ resulting in a line of important findings. For example, crystalline ice growth on $\mathrm{Pt}(111)$ is found to follow the Stranski-Krastanov (SK) growth mode, starting as layer-by-layer followed by threedimensional (3D) island growth, with the transition taking place at a critical thickness of 1 BL (bilayer) in the $\sqrt{39}$ $\times \sqrt{39} R 16.1^{\circ}$ (RT39) phase. ${ }^{3,4}$ Ice layers have been investigated on the $\mathrm{Ru}(0001)$ (Ref. 5) and $\mathrm{Rh}(111)$ (Ref. 6) surfaces, and formation of two-dimensional (2D) clusters has also been explored on $\operatorname{Pd}(111)$ at submonolayer water coverage. ${ }^{7}$ In contrast to these cases, water on the $\mathrm{Au}(111)$ surface has long been known to exhibit hydrophobic behavior. $^{8}$

Despite the numerous studies of water wetting and ice growth, a microscopic understanding of water wettability is still lacking. Macroscopically, a wetting surface is defined as having a contact angle $\theta<90^{\circ}$. At the molecular level, there are no concrete arguments to explain the observed behavior

${ }^{a)}$ Electronic mail: shmeng@ deas.harvard.edu. of water, even for the simplest cases, on the close-packed noble and transition metal surfaces. All studies in which water adsorbs as intact molecules on these surfaces gave much lower adsorption energies ${ }^{9,10}$ than the bulk ice formation en$\operatorname{ergy}\left[E_{\text {ice }} \sim 0.67 \mathrm{eV} / \mathrm{H}_{2} \mathrm{O}\right.$ (Refs. 11 and 12)]. Based on a simple energetic argument, "nonwetting" behavior is expected under these conditions. ${ }^{12}$ However, experiments showed that water does wet these surfaces. ${ }^{3-7,13}$ Alternative structures, such as partially dissociated water molecules, have been proposed to explain water wetting on $\mathrm{Ru}(0001){ }^{12}$ On the other hand, subsequent studies have revealed that water dissociation on $\mathrm{Ru}(0001)$ is hindered by an activation barrier of $0.5-0.62 \mathrm{eV}$ before desorption, ${ }^{14-16}$ making water dissociation an unlikely explanation for the observed wetting. Indeed, recent observations confirm that molecular $\mathrm{D}_{2} \mathrm{O}$ overlayers do wet the $\mathrm{Ru}(0001)$ surface.$^{17}$ Moreover, it is further confirmed that a wetting layer and subsequent $3 \mathrm{D}$ crystalline islands form on $\mathrm{Pt}(111), \mathrm{Pd}(111)$, and $\mathrm{Ru}(0001)$ surfaces. ${ }^{18-20}$ The controversy therefore remains despite the fundamental significance in understanding surface wetting phenomena at the molecular level.

In this work, we construct a microscopic theory of wetting and ice growth on the close-packed metal surfaces. Our theory provides a natural way to understand water wetting on these surfaces without having to assume partial dissociation of water molecules. We argue that the commonly employed criterion in literature, that is, a comparison of surface adsorption energy to the formation energy of bulk ice, which does not take into account growth kinetics and substrate effects, is inappropriate and can even be misleading. Instead, we propose that water wets the surface whenever its overlayer has a 


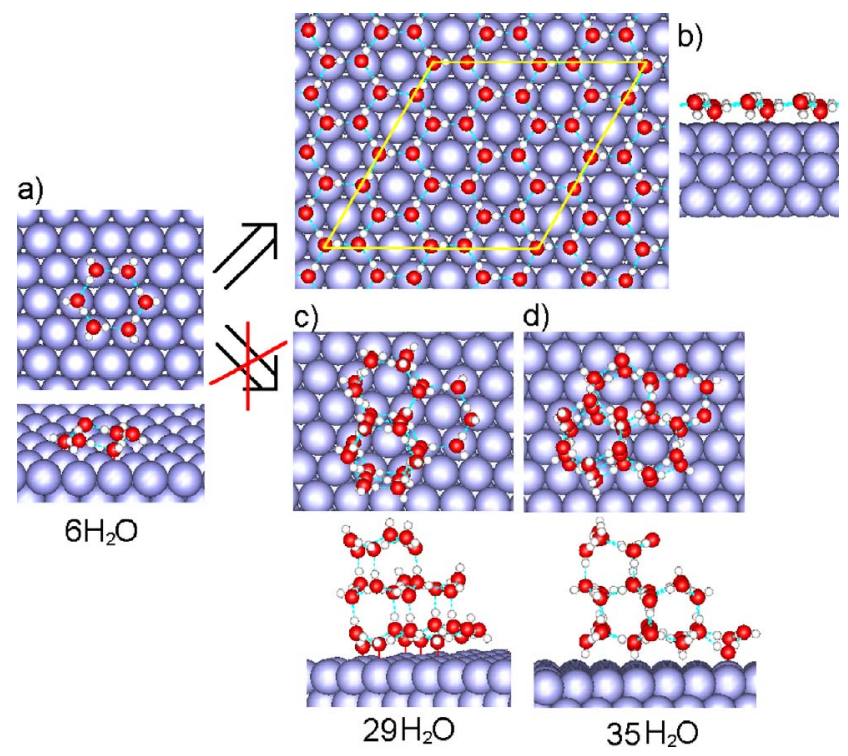

FIG. 1. (Color online) Optimized geometries (top and side views) on the $\mathrm{Pt}(111)$ surface of a (a) water hexamer, (b) bilayer, (c) $29 \mathrm{H}_{2} \mathrm{O} 3 \mathrm{D}$ cluster, and (d) $35 \mathrm{H}_{2} \mathrm{O} 3 \mathrm{D}$ cluster. The solid lines indicate the $(6 \times 6)$ supercell; the dashed lines denote the $\mathrm{H}$ bonds.

larger adsorption energy than 3D clusters. This criterion explains successfully water wetting on $\mathrm{Pt}, \mathrm{Ru}$, and $\mathrm{Rh}$ surfaces, as well as the hydrophobic nature of Au. Using the same criterion, we also explain the growth mechanism on $\operatorname{Pt}(111)$ being the SK type.

Our study is based on first-principles calculations within density functional theory (DFT), employing VASP ${ }^{21}$ with the projector-augmented-wave method ${ }^{22}$ to describe the electronnucleus interaction and the PBE functional for electron exchange-correlation effects. ${ }^{23}$ We use slabs to represent the surfaces and computational parameters that describe accurately both the structure and energetics of ice layers and clusters. ${ }^{24}$ For example, the structural properties of bulk ice in the hexagonal Ih phase are well reproduced: the in-plane lattice constant is $4.39 \AA$ and the out-of-plane one is $7.39 \AA$, compared to the experimental values of 4.496 and $7.323 \AA$, respectively. ${ }^{25}$ The cohesive energy is $0.673 \mathrm{eV}$ per water, in agreement with other theoretical [0.66 eV (Refs. 11 and 12)] and experimental results [0.63 eV (Ref. 26)].

We consider first the behavior of the initial water overlayer on metal surfaces. Water can form either a strained epitaxial overlayer (wetting) or 3D clusters without strain (nonwetting). The first complete water layer closest to the metal surface consists of water molecules arranged in two planes (thus called a bilayer) and connected to form puckered hexagonal networks resembling those in bulk ice, see Fig. 1(b). The ice lattice in adsorbed clusters or commensurate bilayers typically adjusts to that of the substrate due to the flexibility of $\mathrm{H}$ bonds compared with the metal bonding. This has been verified quantitatively in experiments for small clusters on $\operatorname{Ag}(111)$ (Ref. 27) and for overlayers on $\mathrm{Ru}(0001)$ (Ref. 5) and $\mathrm{Rh}(111){ }^{6}$ We therefore consider commensurate overlayers only in the following discussion for convenience. We have calculated the energies of the relevant adsorption structures on four representative surfaces, $\mathrm{Pt}(111), \mathrm{Ru}(0001)$, $\mathrm{Rh}(111)$, and $\mathrm{Au}(111)$. Only small clusters containing several up to a few tens of atoms or molecules are critical in growth kinetics. ${ }^{28}$ Hence, we consider here only small clusters in sizes of 6-35 water molecules.

On the $\operatorname{Pt}(111)$ surface, water can form three different phases, designated RT39, RT37 $\left(\sqrt{37} \times \sqrt{37} R 25.3^{\circ}\right)$, and RT3 $\left(\sqrt{3} \times \sqrt{3} R 30^{\circ}\right)$. The complete RT39 bilayer grows in a SK mode with a critical thickness of $1 \mathrm{BL}$; this phase transforms into the RT3 structure at coverages $>5 \mathrm{BL}$ or under beam exposure. ${ }^{3}$ We find the adsorption energies $\left(E_{\mathrm{ads}}\right)$ of the RT39 and RT3 phases to be 0.55 and $0.51 \mathrm{eV}$, close to but lower than previous calculations; ${ }^{9}$ in view of the fact that those calculations employed less accurate approaches to describe the electron-ion interactions and the electron exchange-correlation effects, such minor differences are not unexpected. The adsorption energy used throughout this work is defined as the energy difference per water molecule between the adsorption system and that of separated substrate and isolated water molecules. The energy difference between RT39 and RT3 explains why the former phase is preferred for the first bilayer, as observed in experiments. 3,4 However, both are much lower than $E_{\text {ice }}=0.67 \mathrm{eV}$, and therefore might not account for wetting based on energetic arguments exclusively with the formation energy of bulk ice as the reference.

For water clusters, we considered first a prototypical hexamer structure with all water molecules lying flat, Fig. $1(\mathrm{a})$, which has $E_{\mathrm{ads}}=0.53 \mathrm{eV}$. For larger clusters, we considered a 2D structure formed by three connected hexamers in a triangular shape, which has $E_{\text {ads }}=0.54 \mathrm{eV}$, essentially the same as the single hexamer ring. Water molecules lying flat will significantly enhance the adsorption energy of $2 \mathrm{D}$ clusters. $^{7,29}$ The 3D clusters are formed by stacking three bilayers, each consisting of a number of hexamer units in each bilayer [3, 2, and 1 in the first, second, and third bilayers, counting from the interface for the $29 \mathrm{H}_{2} \mathrm{O}$ cluster, Fig. 1(c); 4, 3, and 1 in the first, second, and third bilayers for the $35 \mathrm{H}_{2} \mathrm{O}$ cluster, Fig. 1(d)]. The 35-molecule cluster exhibits poor contact with the surface (forming fewer $\mathrm{Pt}-\mathrm{O}$ bonds), and is therefore a mismatch and proton-disordered cluster. These 3D clusters have adsorption energies of 0.470 and $0.473 \mathrm{eV}$, respectively, substantially lower than those of the water hexamer, 2D clusters, and bilayers. Changing water orientation and cluster configuration results in adsorption energy changes less than $0.01 \mathrm{eV}$. Comparing the formation energy of $3 \mathrm{D}$ clusters and the bilayer, we conclude that the formation of a complete bilayer is preferred over 3D clustering. This constitutes a natural explanation for the wetting behavior observed in experiments. ${ }^{3}$

Similar results are obtained for water on $\mathrm{Ru}(0001)$ and $\mathrm{Rh}(111)$. The complete $2 \mathrm{D}$ bilayer on $\mathrm{Ru}(0001)$ has an adsorption energy of $0.50 \mathrm{eV}$, while large clusters $\left(29 \mathrm{H}_{2} \mathrm{O}\right)$ have adsorption energies of $\sim 0.43 \mathrm{eV}$ only. The energy difference of $0.07 \mathrm{eV}$ indicates that forming a complete 2D bilayer is more favorable than 3D clustering on $\mathrm{Ru}(0001)$, suggesting wetting behavior, without having to invoke dissociation of the water overlayers, consistent with experimental observations. ${ }^{17}$ Similarly, the complete 2D bilayer and 3D clusters on $\mathrm{Rh}(111)$ have adsorption energies of 0.52 and $0.46 \mathrm{eV}$, respectively. Consequently, water prefers to form 


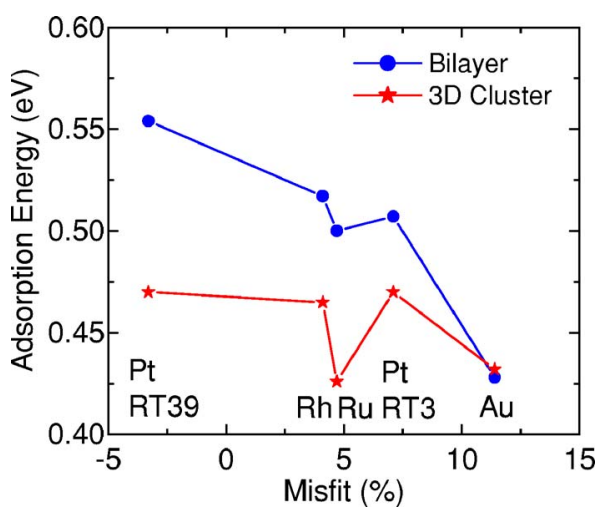

FIG. 2. (Color online) Adsorption energy (eV per water molecule) of the water bilayer (circles) and 3D clusters (stars) on several metal surfaces vs lattice misfit compared to the ideal ice lattice.

2D overlayers rather than $3 \mathrm{D}$ clusters for the first bilayer on $\mathrm{Rh}(111)$ as well. ${ }^{30}$

Since the binding energy of water molecules, both for the first layer and for small finite clusters, on $\mathrm{Pt}, \mathrm{Ru}$, and $\mathrm{Rh}$ is systematically lower than the formation energy in bulk ice, these represent metastable structures compared to large 3D crystalline water droplets. Any large 3D clusters have to grow either on top of the existing first layer or from smaller 3D clusters, which are less stable than the first water layer and not likely to form. Thus, the absence of large 3D crystalline water droplets on bare metal surfaces in experiments indicates that there exist kinetic barriers which block largecluster formation. In addition, a smaller barrier for water diffusion on metal surfaces $(0.1-0.3 \mathrm{eV})$ than interlayer exchange would also favor the first-layer wetting of water on these surfaces.

Water on $\mathrm{Au}(111)$ shows a different behavior. For example, a 3D $29 \mathrm{H}_{2} \mathrm{O}$ cluster has $E_{\text {ads }}=0.432 \mathrm{eV}$, slightly higher than the bilayer adsorption energy of $0.428 \mathrm{eV}$. Therefore 3D islands are as (or slightly more) favored as flat bilayers on $\mathrm{Au}(111)$. This explains the experimental observation of the hydrophobic nature of the Au(111) surface. ${ }^{8}$ To explain this behavior, we invoke first the inert chemical character of Au: a single water molecule has an adsorption energy as low as $0.10 \mathrm{eV}$ on $\mathrm{Au}(111)$. A second important factor is strain. The large misfit (11.4\%) between $\mathrm{Au}(111)$ and ice Ih (0001) further lowers the adsorption energy by as much as $0.07 \mathrm{eV} / \mathrm{H}_{2} \mathrm{O}$. The strain effect is evident in the structural relaxation of water clusters: the H-bond length (OO distance) in the water clusters on $\mathrm{Au}(111)$ is $2.753 \AA$, identical to that in bulk ice, but this is smaller than the $\mathrm{Au}(111)$ lattice constant by $9.3 \%$, a situation very different from that on $\mathrm{Ag}$ (Ref. 27) and $\operatorname{Pd}(111)$ surfaces. ${ }^{7}$ The water bilayer and cluster formation energies on these substrates are plotted in Fig. 2 as a function of the lattice misfit between the substrates and ice, from which it is clear that water bilayers grow on $\mathrm{Pt}, \mathrm{Rh}$, and $\mathrm{Ru}$, but 3D clusters form on $\mathrm{Au}(111)$.

An important issue is what happens above $1 \mathrm{BL}$ coverage on the wetting surfaces. We must compare the formation energy of a water bilayer with that of 3D clusters, both on the ice-covered substrate. However, when the ice film is thick enough, water growth on the substrate resembles that

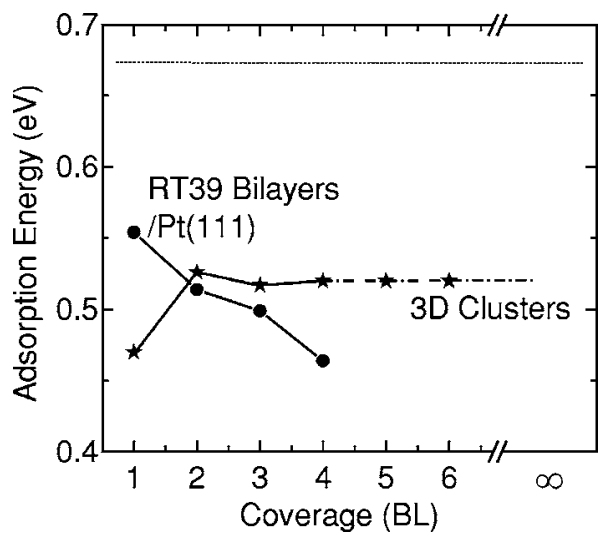

FIG. 3. Adsorption energy of water multilayers within RT39 structure on $\mathrm{Pt}(111)$ as a function of water coverage showing SK growth mode. The stars indicate the 3D ice cluster formation energies (see text for details). The dashed line at $0.67 \mathrm{eV}$ indicates the bulk ice formation energy.

on an ice surface. We find that beyond a 3 BL thickness of ice on metal surface, the single water adsorption energy approaches its corresponding value on free ice surface. Thus the cluster formation energy is almost the same as that on free ice surface beyond $3 \mathrm{BL}$, where the influence of the metal substrate is negligible. Based on this observation, we calculate the adsorption energy of ice clusters on the free ice surface to represent that on water-covered substrates for coverages $>3 \mathrm{BL}$. As the reference state, we use the ideal ice lattice without strain because the strain effect is small as shown before. To represent the naturally occurring ice surface, ice Ih (0001), two structures are considered for the direction of $\mathrm{OH}$ groups that connect adjacent water bilayers along the surface normal direction $z$. The first represents a proton-ordered surface with all interbilayer $\mathrm{OH}$ groups pointing up, which could exist for thicknesses up to a few layers on a surface due to the influence of the surface potential. ${ }^{31}$ The second represents the proton-disordered ice surfaces with the interbilayer $\mathrm{OH}$ groups pointing both up and down to eliminate the total dipole moment along $z$. The disordered orientation of water within the 2D bilayer, which also occurs naturally, is modeled by using large supercells containing 32 water molecules per bilayer.

A $(2 \times 2)$ supercell and 6 BL thick ice slab, consisting of $48 \mathrm{H}_{2} \mathrm{O}$ totally, was used to model the proton-ordered ice surface. On this ice surface, the complete bilayer adsorption energy is $0.68 \mathrm{eV}$, close to the bulk ice formation energy $(0.67 \mathrm{eV})$. However, the water cluster adsorption energy is much lower. For the single hexamer, as well as for a pair of hexamers one on top of the other, this energy is $0.57 \mathrm{eV}$. On the $6 \mathrm{BL}(2 \times 2)$ proton-disordered ice slab, qualitatively similar results are obtained. The complete bilayer has an adsorption energy of $0.66 \mathrm{eV}$ while the single hexamer cluster and the stacked pair of hexamers have adsorption energies of 0.52 and $0.51 \mathrm{eV}$, respectively. The adsorption energies on the proton-disordered surface are smaller than those on proton-ordered surface because of enhanced intermolecular dipolar coupling in the latter. Calculations using larger supercells $(4 \times 4)$ and larger clusters (with 26 and 35 waters) did not give any substantial differences. 
Based on these results, we consider next ice multilayer growth on metal surfaces. Let us take Pt(111) as an example. Figure 3 shows the adsorption energy of the RT39 multilayers and 3D clusters as a function of water coverage on $\mathrm{Pt}(111)$. On the Pt(111) surface, continuous growth of RT39 water bilayers leads to decreasing adsorption energy with coverage, from $0.55 \mathrm{eV}$ of the first bilayer to $0.46 \mathrm{eV}$ of the $4 \mathrm{BL}$ structure. However, the formation energy of $3 \mathrm{D}$ water clusters on bare or ice-covered $\mathrm{Pt}(111)$ is in the range of $0.47-0.52(0.57) \mathrm{eV}$ assuming ice clusters on protondisordered (ordered) ice surface for coverages $>3 \mathrm{BL}$. Comparing with the cluster formation energy, we find a crossover at around $2 \mathrm{BL}$ coverage, indicating that $3 \mathrm{D}$ cluster formation is preferred at this coverage and beyond; therefore, water follows the SK growth mode with a critical thickness of 1 $\mathrm{BL}$ on the RT39/Pt(111) surface. This agrees well with experimental observations that the critical thickness is $1 \mathrm{BL}$ (Ref. 3) and with the hydrophobic nature of the first RT39 bilayer. ${ }^{4,18}$ Recently similar behavior is also observed for water/Pd(111) (Ref. 18) and water/Ru(0001): ${ }^{19,20}$ the first layer wets the bare metal surface and 3D clusters form subsequently on the first water layer, indicating a hydrophilic metal surface and a hydrophobic first water layer upon it. The same mechanism as that for water/Pt(111) may apply for water/Pd and water/Ru, i.e., the first water layer has a special registry with the metal surface facilitating local 3D cluster formation upon it and blocking subsequent overlayer formation. Moreover, the wetting multilayer grown at low temperature $(<130 \mathrm{~K})$ might suggest that multilayers with local ordering ${ }^{18}$ are metastable phases and will transform into 3D clusters upon annealing, very similar to the quantum growth phenomena of flat Ag layers on $\mathrm{GaAs}(110) .^{32}$ Such an investigation is under way.

Finally, we note that the criterion established here through extensive DFT studies of a large number of model systems is in fact conceptually intuitive and simple, as speculated in an earlier attempt to resolve the puzzle on water adsorption on $\mathrm{Ru}(0001) .^{15}$

This work was supported in part by NSF (Grant No. DMR-0606485), and by DOE (CMSN Grant Nos. DE-FG0205ER46226 and DE-FG02-05ER46209, and the Division of Materials Sciences and Engineering, Office of Basic Energy Sciences, DOE, under Contract No. DE-AC05-00OR22725 with Oak Ridge National Laboratory, managed by UTBattelle, LLC). The calculations were performed at ORNL's Center for Computational Sciences.

\footnotetext{
${ }^{1}$ P. A. Thiel and T. E. Madey, Surf. Sci. Rep. 7, 211 (1987).

${ }^{2}$ M. A. Henderson, Surf. Sci. Rep. 46, 1 (2002).

${ }^{3}$ S. Haq, J. Harnett, and A. Hodgson, Surf. Sci. 505, 171 (2002).

${ }^{4}$ G. A. Kimmel, N. G. Petrik, Z. Dohnalek, and B. D. Kay, Phys. Rev. Lett. 95, 166102 (2005).
}

${ }^{5}$ D. L. Doering and T. E. Madey, Surf. Sci. 123, 305 (1982).

${ }^{6}$ K. D. Gibson, M. Viste, and S. J. Sibener, J. Chem. Phys. 112, 9582 (2000).

${ }^{7}$ J. Cerda, A. Michaelides, M. L. Bocquet, P. J. Feibelman, T. Mitsui, M. Rose, E. Fomin, and M. Salmeron, Phys. Rev. Lett. 93, 116101 (2004).

${ }^{8}$ R. S. Smith, C. Huang, E. K. L Wong, and B. D. Kay, Surf. Sci. 367, L13 (1996).

${ }^{9}$ S. Meng, E. G. Wang, and S. W. Gao, Phys. Rev. B 69, 195404 (2004).

${ }^{10}$ A. Michaelides, A. Alavi, and D. A. King, Phys. Rev. B 69, 113404 (2004).

${ }^{11}$ D. R. Hamann, Phys. Rev. B 55, R10157 (1997).

${ }^{12}$ P. J. Feibelman, Science 295, 99 (2002).

${ }^{13}$ H. Ogasawara, B. Brena, D. Nordlund, M. Nyberg, A. Pelmenschikov, L. G. M. Pettersson, and A. Nilsson, Phys. Rev. Lett. 89, 276102 (2002).

${ }^{14}$ S. Meng, E. G. Wang, C. Frischkorn, M. Wolf, and S. W. Gao, Chem. Phys. Lett. 402, 384 (2005).

${ }^{15}$ A. Michaelides, A. Alavi, and D. A. King, J. Am. Chem. Soc. 125, 2746 (2003).

${ }^{16}$ K. Andersson, A. Nikitin, L. G. M. Pettersson, A. Nilsson, and H. Ogasawara, Phys. Rev. Lett. 93, 196101 (2004).

${ }^{17}$ N. S. Faradzhev, K. L. Kostov, P. Feulner, T. E. Madey, and D. Menzel, Chem. Phys. Lett. 415, 165 (2005).

${ }^{18}$ G. A. Kimmel, N. G. Petrik, Z. Dohnalek, and B. D. Kay, J. Chem. Phys. 126, 114702 (2007).

${ }^{19}$ S. Haq and A. Hodgson, J. Phys. Chem. C 111, 5946 (2007).

${ }^{20}$ T. Kondo, H. S. Kato, M. Bonn, and M. Kawai, J. Chem. Phys. 126, 181103 (2007).

${ }^{21}$ G. Kresse and J. Hafner, Phys. Rev. B 47, 558 (1993).

${ }^{22}$ P. E. Blöchl, Phys. Rev. B 50, 17953 (1994).

${ }^{23}$ J. P. Perdew, K. Burke, and M. Ernzerhof, Phys. Rev. Lett. 77, 3865 (1996).

${ }^{24}$ Slab models with three layers of metal atoms and very large surface supercells $(6 \times 6)$ (or RT39) are used to represent the metal surfaces. Thicker slabs do not influence the results significantly. For example, the single water binding energy is $0.120,0.131,0.103$, and $0.109 \mathrm{eV}$, respectively, on a slab consisting of 3, 5, 7, and 9 layers of the $\mathrm{Au}(111)$ surface. The variation of $\pm 0.01 \mathrm{eV}$ is within the typical accuracy of DFT and is smaller than the energy differences discussed in relation to water clusters. The typical thickness of the vacuum layer that separates adjacent slabs is $10 \AA$, in some case reaching $16 \AA$, depending on the water cluster height. The cutoff for the plane-wave basis is $400 \mathrm{eV}$ and a single reciprocalspace point was used. During geometrical optimization, atoms belonging to the bottom layer of the slab are fixed at their respective bulk positions, while all the other surface atoms and the water molecules are allowed to relax fully. Structural optimization is considered converged when the residual forces on atoms have magnitude less than $0.05 \mathrm{eV} / \AA$. A stricter force criterion is not necessary in this study. For instance, further relaxation of a water bilayer on $\mathrm{Ru}(0001)$ using a force convergence criterion of $0.01 \mathrm{eV} / \AA$, results in a lowering of the total energy by $9 \times 10^{-4} \mathrm{eV}$ without apparent structural changes, which makes no difference whatsoever in the relative energy comparisons.

${ }^{25}$ V. R. Brill and A. Tippe, Acta Crystallogr. 23, 343 (1967).

${ }^{26}$ E. Whalley, in The Hydrogen Bond, edited by P. Schuster, G. Zundel, and C. Sandorfy (North-Holland, Amsterdam, 1976), Vol. 3, pp. 1425-1470.

${ }^{27}$ K. Morgenstern and J. Nieminen, Phys. Rev. Lett. 88, 066102 (2002).

${ }^{28}$ U. Gasser, E. R. Weeks, A. Schofield, P. N. Pusey, and D. A. Weitz, Science 292, 258 (2001).

${ }^{29}$ S. Haq, C. Clay, G. R. Darling, G. Zimbitas, and A. Hodgson, Phys. Rev. B 73, 115414 (2006).

${ }^{30}$ A. Beniya, S. Yamamoto, K. Mukai, Y. Yamashita, and J. Yoshinobu, J. Chem. Phys. 125, 054717 (2006).

${ }^{31}$ J. Braun, A. Glebov, A. P. Graham, A. Menzel, and J. P. Toennies, Phys. Rev. Lett. 80, 2638 (1998).

${ }^{32}$ H. B. Yu, C. S. Jiang, P. Ebert, X. D. Wang, J. M. White, Q. Niu, Z. Y. Zhang, and C. K. Shih, Phys. Rev. Lett. 88, 016102 (2002). 